\title{
Euclidean Nonlinear Classical Field Equations with Unique Vacuum
}

\author{
Jeffrey Rauch ${ }^{1 \star}$ and David N. Williams ${ }^{2}$ \\ ${ }^{1}$ Department of Mathematics and \\ 2 Randall Laboratory of Physics, The University of Michigan, Ann Arbor, Michigan 48109, USA
}

\begin{abstract}
We study the real, Euclidean, classical field equation$$
\left(\mu^{2}-\Delta\right) \varphi+\lambda F(\varphi)=f, \quad \mu^{2}>0,
$$

where $\varphi: \mathbb{R}^{d} \rightarrow \mathbb{R}$ is suitably small at infinity. We study existence and regularity assuming that $\lambda \geqq 0, F \in C^{\infty}(\mathbb{R})$, and $a F(a) \geqq 0 \forall a \in \mathbb{R}$. These hypotheses allow strongly nonlinear $F$ and nonunique solutions for $f \neq 0$. When $F^{\prime} \geqq 0$, we prove uniqueness, various contractivity properties, analytic dependence on the coupling constant $\lambda$, and differentiability in the external source $f$. For applications in the loop expansion in quantum field theory, it is useful to know that $\varphi$ is in the Schwartz class $\mathscr{P}$ whenever $f$ is, and we provide a proof of this fact. The technical innovations of the problem lie in treating the noncompactness of $\mathbb{R}^{d}$, the strong nonlinearity of $F$, and the polynomial weights in the seminorms defining $\mathscr{S}$.
\end{abstract}

\section{Introduction}

It is well known [1] that the tree approximation to the first functional derivative of the time-ordered, connected generating functional of a boson quantum field theory obeys the classical field equation with an external source. The tree approximation to the connected generating functional is an infinite formal sum in powers of the coupling constant, over Feynman graphs with no loops. It is the zeroth order term in the loop expansion, which is a formal power series expansion in $h c$.

The same correspondence holds in the Euclidean version of boson field theory, where the time-ordered generating functional is replaced by the Laplace transform of the interacting Euclidean measure on $\operatorname{Re} \mathscr{S}^{\prime}$. We refer to the Laplace transform rather than the Fourier transform in order to arrange for a real classical field with the better sign of the coupling constant.

We want to discuss the classical field equation itself in this paper, and not how it emerges from the limit $\hbar c=0$; but since it was the initial motivation, we describe

* Work supported in part by the NSF under Grant No. MCS 7701748 
the correspondence. Let

$$
\begin{aligned}
\mathscr{E}(f) & =\exp [L(f) / \hbar c] \\
& =N^{-1} \int_{\mathbb{R}_{\mathcal{S}} \mathscr{S}^{\prime}} \exp \left\{-(\hbar c)^{-1} \int[\lambda V(\phi)-\phi f] d x\right\} d \mu_{G}(\phi),
\end{aligned}
$$

where $f \in \operatorname{Re} \mathscr{S}\left(\mathbb{R}^{d}\right), d \mu_{\mathrm{G}}$ is the Gaussian measure for the free, Euclidean scalar field of mass $m$, and $V(\phi)$ is a local interaction, a function of $\phi \in \operatorname{Re} \mathscr{S}^{\prime}\left(\mathbb{R}^{d}\right)$, including renormalization counterterms. The normalization constant $N$ is chosen to make $\mathscr{E}(0)=1$. Of course, the r.h.s. is a formal object. If we make a formal power series expansion of $\delta L / \delta f(x)$ in powers of $\hbar c$, and call the zeroth order term

$$
\phi_{0}(x)=\frac{\delta L_{0}}{\delta f(x)},
$$

it is a theorem in the formal power series sense that

$$
\left(-\Delta+\mu^{2}\right) \phi_{0}+\lambda F\left(\phi_{0}\right)=f, \quad \mu \equiv m c / \hbar,
$$

where $F=V_{0}^{\prime}$ is the derivative of the interaction evaluated at zero in any explicit $\hbar c$ dependence. In practise, this means that $F$ is the derivative of the naive interaction, without counterterms, because the counterterms, including those coming from Wick ordering, are of higher order in $\hbar c$. The above formula is often derived by a stationary phase argument on the Feynman path integral, in the Minkowski version [1]. We refer to [2] for a sketch of the Euclidean argument, which is straightforward.

This paper is an exposition of techniques for studying the Euclidean classical field equation

$$
\left(\mu^{2}-\Delta\right) \varphi+\lambda F(\varphi)=f, \quad \mu^{2}>0, \quad \lambda \geqq 0 .
$$

We look for real solutions for source functions $f$ in a variety of function spaces, including the Sobolev spaces $H_{s}\left(\mathbb{R}^{d}\right), s \geqq-1$, Lebesgue spaces $L^{p}\left(\mathbb{R}^{d}\right)$, and the Schwartz space $\mathscr{S}$.

An immediate application, discussed in [2], is to higher orders of the loop expansion, where the solutions of the classical equation have a central role.

There are two important classes of Euclidean problems, qualitatively richer than ours, which fall outside the scope of our discussion. One is that of three and lower dimensional problems of static, finite energy, soliton solutions of certain non-Abelian gauge field and other models; the other is that of four and lower dimensional instantons in the Euclidean versions of those field theories. A comprehensive and still fairly up to date reference for these problems is the review article of Jackiw [3]. For us, the classical potential term $V_{0}+\mu^{2} \phi^{2} / 2$ has a single minimum at $\phi=0$, which is equivalent to our conditions $a F(a) \geqq 0$ and $\mu^{2}>0$. For the field equations, these conditions imply the uniqueness of the vacuum solution, that is, the solution with zero external source $f$. In the more complicated problems mentioned above, it is exactly the nonuniqueness of the vacuum which is the heart of their interest. Our simpler problem may have some useful points of contact with such problems. 
Our investigation is divided into four parts: existence, regularity, uniqueness and contraction estimates, and dependence on $\lambda$. Corresponding to this division, we state four main theorems.

We use the real Sobolev spaces $H_{s}\left(\mathbb{R}^{d}\right)=\left\{u \in \mathscr{S}^{\prime}\left(\mathbb{R}^{d}\right):\left(1+|\xi|^{2}\right)^{s / 2}|\hat{u}| \in L^{2}\left(\mathbb{R}^{d}\right)\right\}$. For $s$ a nonnegative integer, this is the space of distributions whose partial derivatives of order less than or equal to $s$ are square integrable.

Theorem 1 (Existence of Solutions). If $F \in C(\mathbb{R})$ and $a F(a) \geqq 0$ for all $a \in \mathbb{R}$, then for any $f \in H_{-1}\left(\mathbb{R}^{d}\right)$ there is at least one $\varphi \in H_{1}\left(\mathbb{R}^{d}\right)$ which is a weak solution of the classical field equation in the sense that $F(\varphi) \in L_{\mathrm{loc}}^{1}$ and $E q$. (1.4) is satisfied in the sense of distributions. In addition, $\varphi F(\varphi) \in L^{1}\left(\mathbb{R}^{d}\right)$ and $F(\varphi) \in H_{-1}\left(\mathbb{R}^{d}\right)$.

If $\varphi$ is such a solution, we have $\forall \eta \in \mathscr{D}\left(\mathbb{R}^{d}\right)$

$$
\int \mu^{2} \eta \varphi+\nabla \eta \cdot \nabla \varphi+\eta F(\varphi) d x=\langle\eta, f\rangle,
$$

where $\langle$,$\rangle is the pairing H_{1} \times H_{-1} \rightarrow \mathbb{R}$. Since $F(\varphi) \in H_{-1}$, this identity extends to all $\eta \in H_{1}\left(\mathbb{R}^{d}\right)$, and $\int \eta F(\varphi)$ is replaced by $\langle\eta, F(\varphi)\rangle$. If $f=0$, we may take $\eta=\varphi$ to obtain

$$
\int \mu^{2} \varphi^{2}+|\nabla \varphi|^{2} d x+\langle\varphi, F(\varphi)\rangle=0 .
$$

Formally, $\langle\varphi, F(\varphi)\rangle "={ }^{\prime \prime} \varphi(x) F[\varphi(x)] d x \geqq 0$ since the integrand is nonnegative. It is not difficult to show that indeed $\langle\varphi, F(\varphi)\rangle \geqq 0$ (the necessary techniques are described in Sect. IV), and it follows from the above identity that $\varphi=0$. Thus $\varphi=0$ is the only solution in the absence of a forcing term. However when $f \neq 0$ there may be several solutions.

Theorem 2 (Regularity of Solutions). In addition to the hypotheses of Theorem 1 assume that $F \in C^{\infty}(\mathbb{R})$. Suppose $\varphi \in H_{1}\left(\mathbb{R}^{d}\right), F(\varphi) \in H_{-1}\left(\mathbb{R}^{d}\right) \cap L_{\mathrm{loc}}^{1}\left(\mathbb{R}^{d}\right)$, and $\varphi$ satisfies the classical field Eq. (1.4).

(i) ( $L^{p}$ regularity) If $1<p \leqq \infty$ and $f \in L^{p}\left(\mathbb{R}^{d}\right)$ then $\varphi \in L^{p}\left(\mathbb{R}^{d}\right)$ and $\mu^{2}\|\varphi\|_{L^{p}}$ $\leqq\|f\|_{L^{p}}$. If $f \in L^{\infty}$ then

$$
\varphi \in \bigcap_{p} W_{\mathrm{loc}}^{2, p}\left(\mathbb{R}^{d}\right) \subset \bigcap_{0<\alpha<1} C^{1+\alpha}\left(\mathbb{R}^{d}\right), \quad \lim _{|x| \rightarrow \infty} \varphi(x)=0,
$$

and $\min \{0, \operatorname{ess} \inf f\} \leqq \mu^{2} \varphi \leqq \max \{0$, ess $\sup f\}$.

(ii) (Smooth regularity) If $f \in C^{k+\alpha}\left(\mathbb{R}^{d}\right) \cap L^{\infty}\left(\mathbb{R}^{d}\right)$ then $\varphi \in C^{k+2+\alpha}\left(\mathbb{R}^{d}\right)$ for any integer $k \geqq 0$ and $\alpha \in(0,1)$. If $f \in \mathscr{S}\left(\mathbb{R}^{d}\right)$ then $\varphi \in \mathscr{S}\left(\mathbb{R}^{d}\right)$.

(iii) ( $H_{s}$ regularity, small $s$ ) If $s \in[-1,1]$ and $f \in H_{s}\left(\mathbb{R}^{d}\right) \cap L^{\infty}$ then $\varphi \in H_{s+2}\left(\mathbb{R}^{d}\right)$.

(iv) (Weighted spaces, small s) For any integer $k$ and $s \in \mathbb{R}$ let $\mathscr{S}_{k, s}$ $=\left\{\eta \in H_{s}\left(\mathbb{R}^{d}\right): x^{\alpha} \eta \in H_{s}\left(\mathbb{R}^{d}\right)\right.$ if $\left.|\alpha| \leqq k\right\}$. If $s \in[-1,1]$ and $f \in \mathscr{S}_{k, s} \cap L^{\infty}$ then $\varphi \in \mathscr{S}_{k, s+2}$. Then

In addition to the above hypotheses assume that the number of dimensions $d \leqq 5$.

(v) ( $H_{s}$ regularity) If $s \geqq-1$ and $f \in H_{s}\left(\mathbb{R}^{d}\right)$ then $\varphi \in H_{s+2}\left(\mathbb{R}^{d}\right)$.

(vi) (Weighted spaces) If $k \geqq 0$ is an integer, $s \in[-1, \infty)$, and $f \in \mathscr{T}_{k, s} \cap L^{\infty}$, then $\varphi \in \mathscr{S}_{k, s+2}$.

Remarks. 1. We do not know if (v) and (vi) are true without the restriction $d \leqq 5$.

2. For each result except the $\mathscr{S}$ result in (iii) $F$ need only be differentiable to finite order. For example $F \in C(\mathbb{R})$ suffices for (i), and $F \in C^{1}(\mathbb{R})$ suffices for (iii) and (iv) while $F \in C^{s+1}(\mathbb{R})$ suffices for (v) and (vi). 
3. If $f$ and $F$ are real analytic and $f \in L^{\infty}$, then Hopf's theorem [4] implies that $\varphi$ is a real analytic.

4. If $f \in C^{\infty}\left(\mathbb{R}^{d}\right) \cap L^{\infty}$ then $f \in \bigcap_{k} C^{k+\alpha}\left(\mathbb{R}^{d}\right)$ so (iii) implies that $\varphi \in \bigcap_{k} C^{k+\alpha}\left(\mathbb{R}^{d}\right)$ $=C^{\infty}\left(\mathbb{R}^{d}\right)$.

Theorem 3 (Contraction Estimates). Suppose that $F \in C(\mathbb{R}), F(0)=0$, and $F$ is nondecreasing. For $j=1,2$ let $\varphi_{j}$ be solutions, in the sense of Theorem 1, of the field Eq. (1.4) with sources $f_{j} \in H_{-1}\left(\mathbb{R}^{d}\right)$. Then,

(i) $\int \mu^{2}\left(\varphi_{1}-\varphi_{2}\right)^{2}+\left\|\left.\left(\varphi_{1}-\varphi_{2}\right)\right|^{2} d x \leqq\left(1+\mu^{-2}\right)^{2}\right\| f_{1}-f_{2} \|_{H_{-1}}^{2}$.

(ii) If for some $p \in(1, \infty] f_{1}$ and $f_{2}$ are in $L^{p}\left(\mathbb{R}^{d}\right)$, then

$\mu^{2}\left\|\varphi_{1}-\varphi_{2}\right\|_{p} \leqq\left\|f_{1}-f_{2}\right\|_{p}$.

Remark. Inequality (i) implies the uniqueness of solutions with a given source in $H_{-1}\left(\mathbb{R}^{d}\right)$.

Theorem 4 (Smooth Dependence on $\lambda, f$ ). In addition to the hypotheses of Theorem 3 assume that $F \in C^{\infty}(\mathbb{R})$ and that $s+2>d / 2$.

(i) (Differentiable $H_{s}$ dependence) For $f \in H_{s}\left(\mathbb{R}^{d}\right)$ and $\lambda \geqq 0$ let $\varphi(\lambda, f) \in H_{s+2}\left(\mathbb{R}^{d}\right)$ be the unique solution of the field Eq. (1.4). The map $(\lambda, f) \mapsto \varphi(\lambda, f)$ is infinitely differentiable on $[0, \infty) \times H_{s}\left(\mathbb{R}^{d}\right)$ with values in $H_{s+2}\left(\mathbb{R}^{d}\right)$.

(ii) (Analytic dependence on $\lambda$ ) Suppose in addition to the above hypotheses that $F$ is real analytic on $\mathbb{R}$. For fixed $f$ the map $\lambda \mapsto \varphi(\lambda, f)$ is real analytic on $[0, \infty)$ with values in $H_{s+2}\left(\mathbb{R}^{d}\right)$, that is, for each $\lambda_{0} \in[0, \infty)$ there exist $\varphi_{n} \in H_{s+2}\left(\mathbb{R}^{d}\right), n=0,1, \ldots$, and $r>0$ such that $\varphi(\lambda, f)=\sum_{0}^{\infty}\left(\lambda-\lambda_{0}\right)^{n} \varphi_{n}$ is convergent for $\left|\lambda-\lambda_{0}\right|<r$.

(iii) (Weighted spaces $\mathscr{H}_{k, s}$ ) Suppose $k$ is a nonnegative integer and that the hypotheses of (i) are in force. Then the map $\left(\lambda_{2} f\right) \mapsto \varphi(\lambda, f)$ is an infinitely differentiable function on $[0, \infty) \times \mathscr{S}_{k, s}$ with values in $\mathscr{S}_{k, s+2}$. If, in addition, $F$ is real analytic on $\mathbb{R}$, then the map $\lambda \rightarrow \varphi(\lambda, f)$ is real analytic on $[0, \infty)$ with values in $\mathscr{S}_{k, s+2}$.

Remarks. $1 . F$ is real analytic means that $F$ is the restriction to $\mathbb{R}$ of a holomorphic function $\tilde{F}$ on an open set $U \subset \mathbb{C}$ with $\mathbb{R} \subset U$.

2. The map $(\lambda, f) \mapsto \varphi(\lambda, f)$ is $C^{k}$ if $F \in C^{s+k+3}(\mathbb{R})$. That is, $F$ need only be differentiable finitely often to ensure that $\varphi$ is. For proof one need only examine closely the proof of Theorem 4 .

A remark is in order about techniques. The existence theorem is made nontrivial by the fact that $F$ may be rapidly increasing at $\infty$. For example, $F(\varphi)$ $=\varphi \exp \varphi$. In such cases (at least for $d \geqq 4$ ) the nonlinear term is in no sense a small perturbation. The associated Minkowski equations $\varphi_{t i}-\Delta_{d-1} \varphi+\mu^{2} \varphi+F(\varphi)=f$ are poorly understood for such strongly nonlinear $F$. Matters are further exacerbated by the fact that $\mathbb{R}^{d}$ is not compact, making degree theoretic ideas more difficult to apply. It is important to realize that the existence and regularity theorems are proved in a generality that permits multiple solutions. In the absence of a source $(f=0)$, the only solution is $\varphi \equiv 0 ; 0$; bowever, multiple solutions may exist for $f \neq 0$. 
The critical parts of the regularity theorem are the $L^{p}$ estimates, which are derived using multipliers in the fashion of Moser [5] and Brézis and Strauss [6]. The unboundedness of $\mathbb{R}^{d}$ creates technical problems here, too. One cannot prove $L^{\infty}$ estimates by taking the limit as $p \mapsto \infty$. The $L^{\infty}$ estimate (which is the most important one) requires a separate argument resting on a Harnack type inequality of Moser. Once solutions have been shown to be bounded, the growth of $F$ at infinity becomes irrelevant; and the regularity theory proceeds without essential difficulty. It is worth noting that, as far as we know, the regularity in $\mathscr{S}_{k, s}$ spaces has not been observed, even in the linear case. The contraction estimates are proved with multipliers in the same manner as the $L^{p}$ estimates.

To study the dependence on $\lambda$ and $f$, we show that for $(\lambda, f) \approx\left(\lambda_{0}, f_{0}\right)$ one can reduce the study of the field equation to solving a nonlinear equation $G(\lambda, \varphi, f)=0$, where $G$ is smooth. When $F^{\prime} \geqq 0$, the implicit function theorem can be invoked to obtain the desired results.

Many of the techniques we use are not new. However, since nontrivial innovations are required in several places, and because of the importance of the classical nonlinear field equations in mathematical physics, we feel that it is worthwhile to set down the basic facts in accessible fashion.

\section{The Existence Theorem}

This section is devoted to a proof of Theorem 1 . Replacing $\lambda F$ by $F$, we may assume, without loss of generality, that $\lambda=1$. For further notational convenience, we often suppress the labels $\mathbb{R}$ and $\mathbb{R}^{d}$ in the names of functions spaces. The argument of $F$ always takes values in $\mathbb{R}$, and those of $\varphi$ and $f$ in $\mathbb{R}^{d}$.

We use Galerkin's method to construct approximate solutions $\varphi_{k}$. For $k=1,2, \ldots$ choose $V_{k} \subset C_{0}^{\infty}\left(\mathbb{R}^{d}\right)$ such that $V_{k} \subset V_{k+1}, \operatorname{dim} V_{k}<\infty$, and $\cup_{k} V_{k}$ is dense in $H_{1}$. We seek $\varphi_{k} \in V_{k}$ such that

$$
\int \mu^{2} w \varphi_{k}+\nabla w \cdot \nabla \varphi_{k}+w F\left(\varphi_{k}\right) d x=\langle w, f\rangle
$$

for all $w$ in $V_{k}$. The solution $\varphi$ is obtained as the limit of a subsequence of the $\varphi_{k}$.

1. Existence of $\varphi_{k}$

Define $T: C_{0}^{\infty}\left(\mathbb{R}^{d}\right) \rightarrow C_{0}(\mathbb{R})$ by

$$
T(\psi)=\left(\mu^{2}-\mathcal{A}\right) \psi+F(\psi) .
$$

The basic estimate is that $\forall \psi \in C_{0}^{\infty}$

$$
(T(\psi), \psi)_{L^{2}}=\int \mu^{2} \psi^{2}+|\nabla \psi|^{2}+\psi F(\psi) d x \geqq \min \left(1, \mu^{2}\right)\|\psi\|_{H_{1}}^{2},
$$

since $\psi F(\psi) \geqq 0$.

Let $\pi_{k}: L^{2}\left(\mathbb{R}^{d}\right) \rightarrow V_{k}$ be orthogonal projection in $L^{2}$. Consider $V_{k}$ as a scalar product space with the $L^{2}$ inner product. Now $f \uparrow V_{k}$ is a continuous linear functional, so there is a unique $f_{k} \in V_{k}$ such that $\forall w \in V_{k}$

$$
\langle w, f\rangle=\left(w, f_{k}\right)_{L^{2}} \text {. }
$$


Equation (2.1) is then equivalent to the equation $\pi_{k} T\left(\varphi_{k}\right)-f_{k}=0$. We consider $\pi_{k}{ }^{\circ} T-f_{k}$ as a continuous vector field in $V_{k}$. Let $\Gamma_{k}$ be the bounded open convex set $\Gamma_{k}=\left\{v \in V_{k}:\|v\|_{H_{1}}<1+\max \left(1, \mu^{-2}\right)\|f\|_{H_{-1}}\right\}$. We show that $\operatorname{deg}\left(\pi_{k} T-f_{k}, \partial \Gamma_{k}, 0\right)=1$ ([7] is a good reference for degree theory), and thereby conclude that $\pi_{k} T-f_{k}$ has a zero in $\Gamma_{k}$. The basic estimate is that for $\psi \in V_{k}$,

$$
\begin{aligned}
\left(\pi_{k} T(\psi)-f_{k}, \psi\right)_{L^{2}} & =(T(\psi), \psi)_{L^{2}}-\left(f_{k}, \psi\right)_{L^{2}} \\
& \left.\geqq \min \left(1, \mu^{2}\right)\|\psi\|_{H_{1}}^{2}-K f, \psi\right\rangle \mid \\
& \geqq \min \left(1, \mu^{2}\right)\|\psi\|_{H_{1}}^{2}-\|f\|_{H_{-1}}\|\psi\|_{H_{1}} \\
& =\min \left(1, \mu^{2}\right)\|\psi\|_{H_{1}}\left[\|\psi\|_{H_{1}}-\max \left(1, \mu^{-2}\right)\|f\|_{H_{-1}}\right] .
\end{aligned}
$$

On $\partial \Gamma_{k}$ this is strictly positive. Therefore $\Phi_{t}=(1-t)\left(\pi_{k} T-f_{k}\right)+t I, 0 \leqq t \leqq 1$, is a homotopy of $\pi_{k} T-f_{k}$ to $I$ which never vanishes on $\partial \Gamma_{k}$, since for $\psi \in \partial \Gamma_{k}$, $\left(\Phi_{t}(\psi), \psi\right)_{L^{2}}>0$. It follows that

$$
\operatorname{deg}\left(\pi_{k} T-f_{k}, \partial \Gamma_{k}, 0\right)=\operatorname{deg}\left(I, \partial \Gamma_{k}, 0\right)=1 .
$$

Thus for each $k$ we may choose $\varphi_{k} \in V_{k}$ satisfying (2.1) and in addition with $\left\|\varphi_{k}\right\|_{H_{1}}$ $<1+\max \left(1, \mu^{-2}\right)\|f\|_{H_{-1}}$. Thus, taking $w=\varphi_{k}$ in (2.1), we obtain the additional estimate that

$$
\begin{aligned}
\int \varphi_{k} F\left(\varphi_{k}\right) & \leqq\left\langle\varphi_{k}, f\right\rangle \leqq\left\|\varphi_{k}\right\|_{H_{1}}\|f\|_{H_{-1}} \\
& \leqq \text { constant independent of } k .
\end{aligned}
$$

\section{Use of Compactness to Extract a Convergent Subsequence}

The Rellich compactness theorem, together with the weak compactness of the unit ball in $H_{1}$, imply that there is a subsequence (still denoted $\varphi_{k}$ ) such that

$$
\begin{array}{ll}
\varphi_{k} \rightarrow \varphi & \text { in } H_{1} \text { weakly. } \\
\varphi_{k} \rightarrow \varphi & \text { in } H_{s}^{\text {loc }} \text { strongly for all } s<1 . \\
\varphi_{k} \rightarrow \varphi & \text { pointwise a.e. }
\end{array}
$$

By Fatou's lemma,

$$
\int \varphi F(\varphi) \leqq \liminf \int \varphi_{k} F\left(\varphi_{k}\right),
$$

and the right hand side is finite by (2.7). Thus, $\varphi F(\varphi) \in L^{1}\left(\mathbb{R}^{d}\right)$. Since $|F(s)| \leqq s F(s)$ $+\max _{|s| \leqq 1}|F(s)|$, this implies that $|F(\varphi)| \leqq \varphi F(\varphi)+\max _{|s| \leqq 1}|F(s)|$, so $F(\varphi) \in L_{\text {loc }}^{1}$.

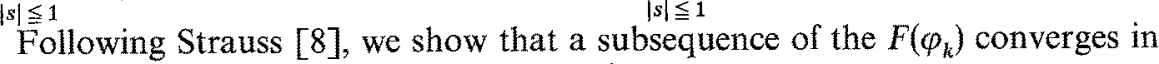
the weak star topology of $L_{\text {loc }}^{1}$ (recall that $\left(L_{\text {loc }}^{1}\right)^{\prime}=L_{\text {compact }}^{\infty}$ ). To do this, it suffices to show that for each $\varepsilon>0$ there is a $\delta>0$ so that $\omega \subset \mathbb{R}^{d}$ and meas $(\omega)<\delta$ imply $\int\left|F\left(\varphi_{k}\right)\right|<\varepsilon$ for all $k$. Given $\varepsilon>0,(2.7)$ permits us to choose $M>0$ so that $2 \int \varphi_{k} F\left(\varphi_{k}\right)<\varepsilon M$ for all $k$. Let $\delta=\varepsilon\left(2 \sup _{|s| \leqq M}|F(s)|\right)^{-1}$. Then $|F(s)| \leqq M^{-1} s F(s)$ 
$+\sup _{|s| \leqq M}|F(s)|$, so if meas $(\omega)<\delta$,

$$
\int_{\omega}|F(\varphi)| \leqq M^{-1} \int \varphi F(\varphi)+\delta \sup _{|s| \leqq M}|F(s)|<\frac{\varepsilon}{2}+\frac{\varepsilon}{2}=\varepsilon .
$$

Thus, passing to a subsequence, again denoted $\varphi_{k}$, we may assume that

$F\left(\varphi_{k}\right) \rightarrow \gamma$ in $L_{\text {loc }}^{1}$ weak star.

It is important to make the identification $\gamma=F(\varphi)$. To do this, observe that for any bounded set $E \subset \mathbb{R}^{d}$ and any $\varepsilon>0$ the pointwise convergence (2.10) together with Egoroff's theorem imply that there is a set $v \subset E$ with meas $(v)<\varepsilon$ and $F\left(\varphi_{k}\right) \rightarrow F(\varphi)$ uniformly on $E \backslash v$. Then $F\left(\varphi_{k}\right) \rightarrow F(\varphi)$ in $L^{\mathbf{1}}(E \backslash v)$ weak star. However by (2.13) $F\left(\varphi_{k}\right) \rightarrow \gamma$ in $L^{1}(E \backslash \nu)$ weak star. Thus $\gamma=F(\varphi)$ a.e. on $E \backslash v$. Since $\varepsilon>0$ was arbitrary, it follows that $\gamma=F(\varphi)$ a.e. in $E$ and therefore almost everywhere in $\mathbb{R}^{d}$.

\section{Passage to the Limit $k \rightarrow \infty$}

Suppose $w \in V_{l}$ for some $l$. Then (2.1) holds provided $k \geqq l$; and we may pass to the limit $k \rightarrow \infty$ in $(2.1)$, since $w \in H_{1}, \varphi_{k}-\varphi$ in $H_{1}, w \in L_{\text {compact }}^{\infty}$ and $F\left(\varphi_{k}\right) \rightarrow F(\varphi)$ in $L_{\text {loc }}^{1}$ weak star. Thus $\forall w \in \cup_{k} V_{k}$,

$$
\int \mu^{2} w \varphi+\nabla w \cdot \nabla \varphi+w F(\varphi) d x=\langle w, f\rangle .
$$

Estimating crudely, we find that $\int|w F(\varphi)| \leqq$ const $\|w\|_{H_{1}}$. Since $\cup_{k} V_{k}$ is dense in $H_{1}$, this implies that $F(\varphi) \in H_{-1}$ and that (2.14) extends by continuity to all $w \in H_{1}$. Since identity (2.14) is equivalent (for $\varphi \in H_{1}$ ) to the field equation (1.4), all the assertions of the existence theorem have been verified.

Remark. Except for the intervention of the local spaces $H_{s}^{\text {loc }}\left(\mathbb{R}^{d}\right)$ and $L_{\text {loc }}^{1}\left(\mathbb{R}^{d}\right)$ in (2.9) and (2.13), this argument is standard. The noncompactness of $\mathbb{R}^{d}$ is only a minor inconvenience here.

\section{Regularity Theorems}

The critical step in the regularity theory is to show that if $f$ is bounded then so is $\varphi$. For classical solutions this is a simple consequence of the maximum principle.

Boundedness of Classical Solutions. If $\varphi \in C^{2}\left(\mathbb{R}^{d}\right)$ is a solution of the classical field equation (1.4) with $f \in L^{\infty}\left(\mathbb{R}^{d}\right) \cap C\left(\mathbb{R}^{d}\right)$ and $|\varphi(x)| \rightarrow 0$ as $|x| \rightarrow \infty$ then $\mu^{2}\|\varphi\|_{L^{\infty}} \leqq\|f\|_{L^{\infty}}$.

Proof. We show that $\mu^{2} \varphi \leqq \max \left\{\sup _{\mathbb{R}^{d}} f, 0\right\}$. The proof that $\mu^{2} \varphi \geqq \min \{\inf f, 0\}$ is similar.

If $\sup \varphi \leqq 0$ we are done. Otherwise there is an $x_{0} \in \mathbb{R}^{d}$ such that $\sup \varphi=\varphi\left(x_{0}\right)>0$. Then $-\Delta \varphi\left(x_{0}\right) \geqq 0$ and $F\left[\varphi\left(x_{0}\right)\right] \geqq 0$, so the field equation (1.4) implies that $\mu^{2} \varphi\left(x_{0}\right) \leqq f\left(x_{0}\right) \leqq \sup f$, and the result is proved.

The problem is to prove a boundedness theorem for weak solutions. The idea of the proof is easy to describe and is inspired by the multipliers of Moser [5] (see 
also Brézis and Strauss [6]). Temporarily ignoring convergence problems, one takes $\eta=|\varphi|^{p-2} \varphi$ in (1.5) to obtain

$$
\int(p-1)|\varphi|^{p-2}|\nabla \varphi|^{2}+\mu^{2}|\varphi|^{p}+|\varphi|^{p-2} \varphi F(\varphi) d x=\int|\varphi|^{p-2} \varphi f
$$

(as in Sect. II we have taken $\lambda=1$ ).

Dropping two positive terms from the left hand side and applying Hölder's inequality to the right with $1 / p+1 / q=1$ yields

$$
\mu^{2} \int|\varphi|^{p} \leqq\|f\|_{L^{p}}\left(\int|\varphi|^{(p-1) q}\right)^{1 / q} .
$$

Since $(p-1) q=p$ we may divide both sides by $\left(\int|\varphi|^{p}\right)^{1 / q}$ to find the estimate $\mu^{2}\|\varphi\|_{L^{p}} \leqq\|f\|_{L^{p}}$.

This is the assertion of Theorem 2(i). We now supply the ideas necessary to make this argument rigorous.

\section{Proof of Part (i) of Theorem 2}

Suppose $1<p<\infty$ and define $\gamma_{n}: \mathbb{R} \rightarrow \mathbb{R}$ by

$$
\gamma_{n}(s)= \begin{cases}|s|^{p-2} s & \text { if } p \geqq 2 \text { and }|s| \leqq n \\ & \text { or } 1<p<2 \text { and } 1 / n \leqq|s| \leqq n, \\ n^{p-1} \operatorname{sign} s & \text { if }|s| \geqq n, \\ n^{2-p_{S}} & \text { if } 1<p<2 \text { and }|s|<1 / n .\end{cases}
$$

Then $\gamma_{n}$ is uniformly Lipschitz continuous so $\varphi \in H_{1}\left(\mathbb{R}^{d}\right)$ implies that $\gamma_{n}(\varphi) \in H_{1}\left(\mathbb{R}^{d}\right)$ and $\nabla \gamma_{n}(\varphi)$ is the element of $L^{2}\left(\mathbb{R}^{d}\right)$ equal to $\gamma_{n}^{\prime}(\varphi) \nabla \varphi$ where $|\varphi|<n$ and $|\varphi| \neq 1 / n$ and equal to zero otherwise (see Lemma 28.1 in [9]). Choose $\psi \in C_{0}^{\infty}(\mathbb{R})$ such that $0 \leqq \psi \leqq 1, \psi(s)=\psi(-s), \psi(0)=1$, and $\psi^{\prime}(s) \leqq 0$ for $s>0$. Define $\eta_{m} \in C_{0}^{\infty}\left(\mathbb{R}^{d}\right)$ by $\eta_{m}(x)=\psi\left(m^{-1}|x|\right)$. If $\varphi \in H_{1}$ is a solution of the field equation we may take $\eta_{m} \gamma_{m}(\varphi)$ as "test function" in (1.5) to obtain

$$
\int \nabla \varphi \cdot \nabla\left[\eta_{m} \gamma_{n}(\varphi)\right]+\mu^{2} \eta_{m} \varphi \gamma_{n}(\varphi)+\eta_{m} \gamma_{n}(\varphi) F(\varphi) d x=\left\langle f, \eta_{m} \gamma_{n}(\varphi)\right\rangle .
$$

Notice that $F(\varphi) \in L_{\text {loc }}^{1}$ and $\eta_{m} \gamma_{n}(\varphi) \in L_{\text {compact }}^{\infty}$ so the pairing $\left\langle\eta_{m} \gamma_{n}(\varphi), F(\varphi)\right\rangle$ is an integral of a nonnegative function and is therefore nonnegative. Similarly, since $f \in L^{p}$ and $\eta_{m} \gamma_{n}(\varphi) \in L_{\text {compact }}^{\infty}$ the pairing on the right of (3.2) is also an integral. Now

$$
\int \nabla \varphi \cdot \nabla\left[\eta_{m} \gamma_{n}(\varphi)\right]=\int \gamma_{n}(\varphi) \nabla \varphi \cdot \nabla \eta_{m}+\int_{|\varphi|<n} \eta_{m} \gamma_{n}^{\prime}(\varphi)|\nabla \varphi|^{2} \geqq \int \gamma_{n}(\varphi) \nabla \varphi \cdot \nabla \eta_{m}
$$

since $\gamma^{\prime} \geqq 0$. Thus, estimating the right hand side of (3.2) by Hölder's inequality and using the fact that $\eta_{m} \gamma_{n}(\varphi) F(\varphi) \geqq 0$ we have

$$
\begin{aligned}
& \int \gamma_{n}(\varphi) \nabla \eta_{m} \cdot \nabla \varphi+\mu^{2} \eta_{m} \gamma_{n}(\varphi) \varphi d x \\
& \quad \leqq\left(\int|f|^{p} \eta_{m} d x\right)^{1 / p}\left(\int\left|\gamma_{n}(\varphi)\right|^{q} \eta_{m} d x\right)^{1 / q},
\end{aligned}
$$

where $1 / p+1 / q=1$. Now $\left|\gamma_{n}(s)\right|^{q} \leqq s \gamma_{n}(s)$ for all $s \in \mathbb{R}$ (they are equal for $1 / n \leqq|s| \leqq n$ ), so the $q$-norm on the right of (3.4) is at most equal to $\left(\int \varphi \gamma_{n}(\varphi) \eta_{m}\right)^{1 / q}$. Using this estimate and rearranging terms yields

$$
\left(\int \varphi \gamma_{n}(\varphi) \eta_{m}\right)^{1 / q}\left[\mu^{2}\left(\int \varphi \gamma_{n}(\varphi) \eta_{m}\right)^{1 / p}-\left(\int|f|^{p} \eta_{m}\right)^{1 / p}\right] \leqq-\int \gamma_{n}(\varphi) \nabla \eta_{m} \cdot \nabla \varphi .
$$


For fixed $n$, the right hand side is $o(1 / m)$ as $m \rightarrow \infty$, and $\varphi \gamma_{n}(\varphi) \eta_{m} \rightarrow \varphi \gamma_{n}(\varphi)$ and $|f|^{p} \eta_{m} \rightarrow|f|^{p}$, both monotonically. The monotone convergence theorem allows us to pass to the limit $m \rightarrow \infty$ in the integrals in (3.5) to conclude that $\int \varphi \gamma_{n}(\varphi)<\infty$ and moreover

$$
\mu^{2} \int \varphi \gamma_{n}(\varphi) \leqq \int|f|^{p}
$$

Letting $n \rightarrow \infty, \varphi \gamma_{n}(\varphi) \lambda|\varphi|^{p}$, so the monotone convergence theorem yields $\mu^{2}\|\varphi\|_{p} \leqq\|f\|_{p}$, which is the desired result.

To derive the $L^{\infty}$ estimate the argument must be modified substantially. Notice that one cannot merely let $p \rightarrow \infty$ since $f$ need not be in any $L^{p}$ space other than $L^{\infty}$, and secondly $\|f\|_{L^{\infty}}$ might be smaller than $\lim _{p \rightarrow \infty}\|f\|_{L^{p}}$. What does immediately follow is that

$$
\mu^{2}\|\varphi\|_{\infty} \leqq \liminf _{p \rightarrow \infty} \mu^{2}\|\varphi\|_{p} \leqq \liminf _{p \rightarrow \infty}\|f\|_{p},
$$

which suffices for many applications. For example, if $f \in L^{\infty} \cap L^{p_{0}}$ for some $p_{0}<\infty$ and ess sup $|f| \rightarrow 0$ as $R \rightarrow \infty$, then the right hand side of (3.6) is equal to $\|f\|_{\infty}$. To $|x|>R$ derive a sup norm estimate for $\varphi$ without the hypothesis that $|f| \rightarrow 0$ at infinity, we use more of the elegant ideas of Moser [5].

Lemma 1. Suppose that $\Omega \subset \mathbb{R}^{d}$ is an open set, $\varphi \in H_{1}^{\mathrm{loc}}(\Omega), F(\varphi) \in L_{1}^{\mathrm{loc}}(\Omega), f \in L_{\mathrm{loc}}^{1}(\Omega)$, and $\left(\mu^{2}-\Delta\right) \varphi+F(\varphi)=f$. Suppose $k: \mathbb{R} \rightarrow \mathbb{R}$ is a nonnegative convex function such that $k(0)=0$ and there is a $\varrho>0$ with $k^{\prime \prime}(s)=0$ if $|s|>\varrho$. Then $\Delta k(\varphi) \geqq-f k^{\prime}(\varphi)$.

Remark. Since $f \in L_{1}^{\text {loc }}$ and $k^{\prime}(\varphi) \in L^{\infty}$, it follows that $f k^{\prime}(\varphi) \in \mathscr{D}^{\prime}$, so the assertion $-\Delta k(\varphi) \leqq f k^{\prime}(\varphi)$ makes sense and is equivalent to the fact that $\int_{\Omega} \nabla \psi \cdot \nabla \mathrm{k}(\varphi)$ $-\psi \mathrm{fk}^{\prime}(\varphi) d x \leqq 0$ for all nonnegative $\psi \in \mathscr{D}\left(\mathbb{R}^{d}\right)$.

Proof. We prove the result assuming that $k \in C^{2}(\mathbb{R})$. The general case follows as in [5] by approximating general $k$ by convex functions satisfying the additional hypothesis. Since $k^{\prime \prime}=0$ for $|s|$ large, $k$ is Lipschitz continuous, so $\varphi \in H_{1}^{\mathrm{loc}} \Rightarrow k(\varphi) \in H_{1}^{\mathrm{loc}} ;$ and we need only show that $\Delta k(\varphi) \geqq-f k^{\prime}(\varphi)$.

By hypothesis

$$
\int \mu^{2} \eta \varphi+\nabla \eta \cdot \nabla \varphi+\eta F(\varphi) d x=\int \eta f d x
$$

$\forall \eta \in H_{1}(\Omega) \cap \mathscr{E}^{\prime}(\Omega)$. Take $\eta=\psi k^{\prime}(\varphi)$ with $\psi \in \mathscr{D}(\Omega), \psi \geqq 0$. Since $\operatorname{sign} k^{\prime}(\varphi)=\operatorname{sign} \varphi$, it follows that $\varphi k^{\prime}(\varphi)$ and $k^{\prime}(\varphi) F(\varphi)$ are nonnegative; so $0 \leqq \int \mu^{2} \eta \varphi+\eta F(\varphi) d x$. Thus

$$
\int \nabla \eta \cdot \nabla \varphi d x \leqq \int \eta f
$$

Evaluating $\nabla \eta$ yields

$$
\int \nabla \psi \cdot \nabla k(\varphi) \leqq \int \psi k^{\prime}(\varphi) f-\psi k^{\prime \prime}(\varphi)|\nabla \varphi|^{2} d x \leqq \int \psi k^{\prime}(\varphi) f,
$$

since $k^{\prime \prime} \geqq 0$. The proof that $-\Delta k(\varphi) \leqq f k^{\prime}(\varphi)$ is complete.

Now suppose that $\varphi$ and $f$ are as in Part (i) of the regularity theorem and that $p=\infty$. Let $k(s)=|s|$; then Lemma 1 implies that $-\Delta|\varphi(s)| \leqq\|f\|_{\infty}$ on $\mathbb{R}^{d}$. Moser's method [5] yields a constant $c>0$ so that for any $x_{0} \in \mathbb{R}^{d}$

$$
\underset{\left|x-x_{0}\right|<1}{\operatorname{ess} \sup }|\varphi| \leqq c\left(\int_{\left|x-x_{0}\right|<2}|\varphi(x)|^{2} d x\right)^{1 / 2} \text {. }
$$


The proof is exactly as in [5]. A more general result is proved in Theorem 8.17 of Gilbarg and Trudinger [10]. Since $\varphi \in L^{2}\left(\mathbb{R}^{d}\right)$ it follows that $\varphi \in L^{\infty}\left(\mathbb{R}^{d}\right)$ and that ess sup $|\varphi(x)| \rightarrow 0$ as $R \rightarrow \infty$. Consequently, $f+F(\varphi) \in L^{\infty}\left(\mathbb{R}^{d}\right)$; and the elliptic $|x|>R$

regularity theorem for the operator $\mu^{2}-\Delta$ implies that $\varphi \in \bigcap_{p>1} W_{\mathrm{loc}}^{2, p}\left(\mathbb{R}^{d}\right)$. In particular, $\varphi$ is continuous. We have proved the last sentence of Theorem 2(i).

We next prove the estimate $\mu^{2} \varphi \leqq \max \{0$, ess $\sup f\}$ for $f \in L^{\infty}$. Let

$$
\Omega=\left\{x \in \mathbb{R}^{d}: \mu^{2} \varphi(x)>\max \{0, \operatorname{ess} \sup f\}\right\} .
$$

By the above assertions $\Omega$ is an open set, which is bounded if ess $\sup f>0$; and in $\Omega$ we have

$$
\begin{aligned}
& \Delta \varphi=\mu^{2} \varphi+F(\varphi)-f \geqq 0, \\
& \lim _{x \rightarrow \partial \Omega} \mu^{2} \varphi(x)=\max \{0, \text { ess sup } f\} .
\end{aligned}
$$

It follows from the classical maximum principle for subharmonic functions that $\mu^{2} \varphi \leqq \max \{0, \operatorname{ess} \sup f\}$ in $\Omega$. Therefore $\Omega$ must be empty, and $\sup \mu^{2} \varphi$ $\leqq \max \{0, \operatorname{ess} \sup f\}$. That $\inf \mu^{2} \varphi \geqq \min \{0$, essinf $f\}$ is proved in the same manner with

$$
\Omega=\left\{x \in \mathbb{R}^{d}: \mu^{2} \varphi(x)<\min \{0, \operatorname{ess} \inf f\}\right\},
$$

and the proof of the first part of the regularity theorem is complete.

\section{Proof of Theorem 2 Part (ii)}

By Part (i) $\varphi \in C^{1+\alpha}$. The result follows from a finite number of applications of the following "bootstrap" lemma.

Lemma 2. If $\varphi \in C^{l+\alpha}$ and $f \in C^{k+\alpha}$ with $k, l$ nonnegative integers, then $\varphi \in C^{2+\min (k, l)+\alpha}$.

Proof of Lemma. Since $\varphi \in C^{l+\alpha}, F(\varphi) \in C^{l+\alpha}$, so $\left(\mu^{2}-\Delta\right) \varphi=f-F(\varphi) \in C^{\min (k, l)+\alpha}$, and the result follows from classical (circa 1900) regularity theorems for $\mu^{2}-4$.

From the lemma, if $l \leqq k, \varphi$ gains two derivatives. If $l>k$, the conclusion is $\varphi \in C^{2+k+\alpha}$. Let $m$ be the unique integer such that $l+2 m \leqq k<l+2(m+1)$. Then $m+1$ applications of the lemma yield $\varphi \in C^{l+2(m+1)+\alpha}$. If $l+2 m=k$ we are done. If $l+2 m<k$ one more application of the lemma yields the desired result, for finitely differentiable $f$.

We now go to the case $f \in \mathscr{S}$. Let $\psi \in H_{1}\left(\mathbb{R}^{d}\right)$ be the unique solution of $\left(\mu^{2}-\Delta\right) \psi$ $=\left(f^{2}+\exp -x^{2}\right)^{1 / 2} \equiv h$. Since $h$ is a positive element of $\mathscr{S}$ it follows that $\psi>0$ and $\psi \in \mathscr{S}$. We show that $\varphi$ is small at infinity by proving $|\varphi| \leqq \psi$. Since both $\varphi$ and $\psi$ are smooth and vanish at infinity, either $\varphi \leqq \psi$ or the positive maximum of $\varphi-\psi$ occurs at some point $x_{0} \in \mathbb{R}^{d}$. The latter alternative cannot occur, for at such an $x_{0}$ one would have $(\varphi-\psi)>0, \Delta(\varphi-\psi) \leqq 0, \varphi \geqq \psi \geqq 0$, so $F(\varphi)>0$ and

$$
\left(\mu^{2}-\Delta\right)(\varphi-\psi)+F(\varphi)=f-\left(f+\exp -x^{2}\right)^{1 / 2}<0,
$$

a contradiction. That $\varphi \geqq-\psi$ is proved similarly. 
Let $B_{r}(x)=\left\{y \in \mathbb{R}^{d}:|y-x|<r\right\}$. Then the estimate $|\varphi| \leqq \psi$ implies that, for any $r$ and $N, \int_{B_{r}(x)}|\varphi|^{2}=O\left(|x|^{-N}\right)$ as $x \rightarrow \infty$.

To prove that $\varphi \in \mathscr{P}$, it suffices to prove that for all $r>0$ and integer $N$ and $s,\|\varphi\|_{H_{s}\left(B_{r}(x)\right)}=O\left(|x|^{-N}\right)$ as $x \rightarrow \infty$. The proof is by induction on $s$. The case $s=0$ has been proved. The basic ingredient is the elliptic estimate:

$$
\|\varphi\|_{H_{s+2}\left(B_{r}(x)\right)} \leqq C(r, s)\left(\|\varphi\|_{H_{s}\left(B_{2 r}(x)\right)}+\|\Delta \varphi\|_{H_{s}\left(B_{2 r}(x)\right)}\right) .
$$

Suppose our assertion is proved for $s \leqq s_{0}$. Then $\|\varphi\|_{B_{s_{0}}\left(B_{2 r}(x)\right)}=O\left(|x|^{-N}\right)$, and from the field equation $\Delta \varphi=\mu^{2} \varphi+F(\varphi)-f$, so $\|\Delta \varphi\|_{H_{s_{0}}\left(B_{2 r}(x)\right)}=O\left(|x|^{-N}\right)$, since $F$ vanishes at the origin; and it follows that $\|\varphi\|_{H_{s+2}\left(B_{r}(x)\right)}=O\left(|x|^{-N}\right)$.

\section{Proof of Theorem 2 Part (iii)}

By Part (i), $\varphi \in L^{\infty}\left(\mathbb{R}^{d}\right)$. Choose $\tilde{F} \in C_{0}^{\infty}(\mathbb{R})$ such that $\tilde{F}(s)=F(s)$ if $|s| \leqq\|\varphi\|_{L^{\infty}}$. Then $F(\varphi)=\tilde{F}(\varphi)$. Now $F$ is Lipschitz continuous and $H_{1}\left(\mathbb{R}^{d}\right)$ is stable under composition with Lipschitz maps so $\tilde{F}(\varphi) \in H_{1}$. Therefore $\left(\mu^{2}-\Delta\right) \varphi=f-F(\varphi) \in H_{s}$ so $\varphi \in H_{s+2}$.

\section{Proof of Part (iv) of Theorem 2}

We require a lemma about the spaces $\mathscr{S}_{k, s}$.

Lemma 3. (i) For all $s, \sigma \in \mathbb{R}$ and integers $k,\left(\mu^{2}-\Delta\right)^{\sigma}$ is an isomorphism of $\mathscr{S}_{k, s+2 \sigma} \rightarrow \mathscr{S}_{k, s}$

(ii) $\varphi \in \mathscr{S}_{k, 1} \cap L^{\infty} \Rightarrow F(\varphi) \in \mathscr{S}_{k, 1}$.

(iii) If $s>d / 2$ then $\varphi \in \mathscr{H}_{k, s} \Rightarrow F(\varphi) \in \mathscr{S}_{k, s}$.

Proof. (i) That $\left(\mu^{2}-\Delta\right)^{\sigma}$ is $1-1$ and continuous from $\mathscr{S}_{k, s+2}$ to $\mathscr{S}_{k, s}$ is elementary. To show that it is onto, suppose $\eta \in \mathscr{T}_{k, s}$ and $\varphi \in H_{s+2}$ satisfies $\left(\mu^{2}-\Delta\right)^{\sigma} \varphi=\eta$. We must show that $\varphi \in \mathscr{T}_{k, s+2 \sigma}$. This is an immediate consequence of the identity $\widehat{x^{\alpha} \varphi}$ $=(-i \partial / \partial \xi)^{\alpha}\left[\left(\mu^{2}+|\xi|^{2}\right)^{-\sigma} \hat{\eta}\right]$.

(ii) Choose $\tilde{F} \in C_{0}^{\infty}(\mathbb{R})$ such that $\tilde{F}(a)=F(a)$ if $|a| \leqq\|\varphi\|_{L^{\infty}}$. Then $F(\varphi)=\tilde{F}(\varphi)$. Since $\varphi \in H_{1}$ and $\tilde{F}$ is Lipschitz it follows that $F(\varphi) \in H_{1}$. In addition, for all $i$, $\left|x_{i} \tilde{F}(\varphi)\right| \leqq c\left|x_{i} \varphi\right|$, where $c=\max \left|\tilde{F}^{\prime}\right|$, so $x_{i} F(\varphi) \in L_{2}$. Similarly $D_{j}\left(x_{i} \tilde{F}(\varphi)\right)=\delta_{i j} \tilde{F}(\varphi)$ $+x_{i} \tilde{F}^{\prime}(\varphi) D_{j} \varphi$, so $\left|D_{j} x_{i} \tilde{F}(\varphi)\right| \leqq|\tilde{F}(\varphi)|+c\left|x_{i} D_{j} \varphi\right|$, and the right hand side is in $L^{2}$, so $x_{i} F(\varphi) \in H_{1}$. This proves (ii) for $k=1$. Analogous arguments yield an inductive proof of the general result.

(iii) The proof of this is exactly the same as the proof of Schauder's lemma [see the proof of Part (v)] so is not short and not illuminating. It is omitted.

To prove Part (iv) we first treat the case $s=-1$; that is, we must show that if $f \in \mathscr{F}_{k,-1}$ then $\varphi \in \mathscr{S}_{k, 1}$. For $k=0$ there is nothing to prove. Consider next the case $k=1$. We must show that $x_{i} \varphi \in H_{1}$ for all $i$. Formally the proof is simple. Take $\eta=x_{i}^{2} \varphi$ in (1.5) to obtain

$$
\begin{aligned}
& \int \mu^{2} x_{i}^{2} \varphi^{2}+\prod\left(x_{i}^{2} \varphi\right) \cdot \nabla \varphi d x+\left\langle x_{i}^{2} \varphi, F(\varphi)\right\rangle \\
& \quad=\left\langle x_{i} \varphi, x_{i} f\right\rangle .
\end{aligned}
$$


Now $\left\langle x_{i}^{2} \varphi, F(\varphi)\right\rangle$ is formally nonnegative, so

$$
\begin{aligned}
\left\|x_{i} \varphi\right\|_{H_{1}}^{2} & \cong \int \mu^{2} x_{i}^{2} \varphi^{2}+\nabla\left(x_{i} \varphi\right) \cdot \nabla\left(x_{i} \varphi\right) d x \\
& =\int \mu^{2} x_{i}^{2} \varphi^{2}+\nabla\left(x_{i}^{2} \varphi\right) \cdot \nabla \varphi d x+\int \varphi^{2} d x \\
& \leqq\left\langle x_{i} \varphi, x_{i} f\right\rangle+\|\varphi\|_{L^{2}}^{2} \\
& \leqq\left\|x_{i} \varphi\right\|_{H_{1}}\left\|x_{i} f\right\|_{H_{-1}}+\|\varphi\|_{L^{2}}^{2},
\end{aligned}
$$

and a bound on $\left\|x_{i} \varphi\right\|_{H_{i}}$ follows.

To make this rigorous, choose $\chi \in C_{0}^{\infty}\left(\mathbb{R}^{d}\right)$ such that $\chi \geqq 0$ and $\chi(0)=1$. Let $\chi_{\varepsilon}(x)$ $=\chi(\varepsilon x)$ and take $\eta=\chi_{\varepsilon}^{2} x_{i}^{2} \varphi$ in (1.5). Then $\left\langle\eta x_{i}^{2} \varphi, F(\varphi)\right\rangle \geqq 0$, and one finds that

$$
\begin{aligned}
& \int \mu^{2} \chi_{\varepsilon}^{2} x_{i}^{2} \varphi^{2}+\nabla\left(\chi_{\varepsilon}^{2} x_{i}^{2} \varphi\right) \cdot \nabla \varphi d x \leqq\left\langle\chi_{\varepsilon} x_{i} \varphi, x_{i} f\right\rangle \\
& \leqq\left\|\chi_{\varepsilon} x_{i} \varphi\right\|_{H_{1}}\left\|\chi_{\varepsilon} x_{i} f\right\|_{H_{-1}},
\end{aligned}
$$

so

$$
\begin{aligned}
\left\|\chi_{\varepsilon} x_{i} \varphi\right\|_{H_{1}}^{2} & \cong \int \mu^{2} \chi_{\varepsilon}^{2} x_{i}^{2} \varphi^{2}+\nabla\left(\chi_{\varepsilon} x_{i} \varphi\right) \cdot \nabla\left(\chi_{\varepsilon} x_{i} \varphi\right) d x \\
& =\int \mu^{2} \chi_{\varepsilon}^{2} x_{i}^{2} \varphi^{2}+\nabla\left(\chi_{\varepsilon}^{2} x_{i}^{2} \varphi\right) \cdot \nabla \varphi d x+\int\left|\nabla\left(\chi_{\varepsilon} x_{i}\right)\right|^{2} \varphi^{2} d x \\
& \leqq\left\|\chi_{\varepsilon} x_{i} \varphi\right\|_{H_{1}}\left\|\chi_{\varepsilon} x_{i} f\right\|_{H_{-1}}+\int\left|\nabla\left(\chi_{\varepsilon} x_{i}\right)\right|^{2} \varphi^{2} d x
\end{aligned}
$$

Let $M=\sup _{0<\varepsilon \leqq 1}\left\|\chi_{\varepsilon} x_{i} f\right\|_{H_{-1}}+\int\left|\nabla\left(\chi_{\varepsilon} x_{i}\right)\right|^{2} \varphi^{2} d x<\infty$. Then

$$
\left\|\chi_{\varepsilon} x_{i} \varphi\right\|_{H_{1}}^{2} \leqq\left(\left\|\chi_{\varepsilon} x_{i} \varphi\right\|+1\right) M,
$$

and it follows that $\chi_{\varepsilon} x_{i} \varphi$ for $\varepsilon \in(0,1]$ is a bounded subset of $H_{1}$. Therefore $x_{i} \varphi=\lim _{\varepsilon \rightarrow 0} \chi_{\varepsilon} x_{i} \varphi$ is in $H_{1}$. This completes the proof for $k=1$ and $s=-1$.

For $k>1$ and $s=-1$ the proof is by induction on $k$. Suppose the result is known for all values less than or equal to $k$. We prove it for $k+1$. That is, if $f \in \mathscr{P}_{k+1,-1}$, we show $\varphi \in \mathscr{S}_{k+1,1}$. Formally, the proof is simple. If $|\alpha|=k+1$ take $\eta=x^{2 \alpha} \varphi$ in (1.5) to obtain

$$
\begin{aligned}
\int \mu^{2} x^{2 \alpha} \varphi^{2}+\nabla x^{2 \alpha} \varphi \cdot \nabla \varphi d x & =\left\langle x^{2 \alpha} \varphi, f\right\rangle-\left\langle x^{2 \alpha} \varphi, F(\varphi)\right\rangle \\
& \leqq\left\langle x^{\alpha} \varphi, x^{\alpha} f\right\rangle .
\end{aligned}
$$

Thus

$$
\begin{aligned}
\left\|x^{\alpha} \varphi\right\|_{H_{1}}^{2} & \cong \int \mu^{2} x^{2 \alpha} \varphi^{2}+\nabla x^{\alpha} \varphi \cdot \nabla x^{\alpha} \varphi d x \\
& =\int \mu^{2} x^{2 \alpha} \varphi^{2}+\nabla x^{2 \alpha} \varphi \cdot \nabla \varphi d x+\int\left|\nabla x^{\alpha}\right|^{2} \varphi^{2} d x \\
& \leqq\left\langle x^{\alpha} \varphi, x^{\alpha} f\right\rangle+c\|\varphi\|_{\mathscr{S}_{k, 1}}^{2} \\
& \leqq\left\|x^{\alpha} \varphi\right\|_{H_{\mathrm{I}}}\left\|x^{\alpha} f\right\|_{H_{-}}+c\|\varphi\|_{\mathscr{S}_{k, 1}}^{2},
\end{aligned}
$$

and a bound for $\left\|x^{\alpha} \varphi\right\|_{H_{1}}$ follows. To make this formal argument rigorous one reasons exactly as in the case $k=1$. This completes the proof when $s=-1$.

If $-1 \leqq s \leqq 1$, the above result implies that $\varphi \in \mathscr{F}_{k, 1}$; so by Part (ii) of Lemma 3 $F(\varphi) \in \mathscr{S}_{k, 1}$; so $\left(\mu^{2}-\Delta\right) \varphi=f-F(\varphi) \in \mathscr{S}_{k, s}$ and $\varphi \in \mathscr{S}_{k, s+2}$ by the first part of the lemma. 


\section{Proof of Theorem 2 Part (v)}

For $s \leqq 1$ the result has already been proved in Part (iv). For $s>1$, (iii) implies that $\varphi \in H_{3}$. Notice that $3>d / 2$ since $d \leqq 5$. The result follows from a finite number of applications of the following bootstrap lemma:

Lemma 4. If $\varphi \in H_{\sigma}\left(\mathbb{R}^{d}\right)$ and $f \in H_{s}\left(\mathbb{R}^{d}\right)$ with $d / 2<\sigma$ then $\varphi \in H_{2+\min (\sigma, s)}\left(\mathbb{R}^{d}\right)$.

Proof. The critical fact is Schauder's lemma, which implies that $F(\varphi) \in H_{\sigma}\left(\mathbb{R}^{d}\right)$. A precise statement is given below.

Schauder's Lemma. Suppose $\sigma \in \mathbb{R}$ and $\sigma>d / 2$.

(i) If $F \in C^{\infty}\left(\mathbb{R}^{k}\right)$ then the map $\left(\eta_{1}, \ldots, \eta_{k}\right) \mapsto F\left(\eta_{1}, \ldots, \eta_{k}\right)$ is a bounded map from $H_{\sigma}\left(\mathbb{R}^{d}\right)^{k} \rightarrow H_{\sigma}\left(\mathbb{R}^{d}\right)$.

(ii) Theabove map is infinitely differentiable. The derivative at $\eta$ is the linear map $\left(h_{1}, \ldots, h_{k}\right) \mapsto \sum_{j} h_{j} D_{j} F(\eta)$.

A proof for $\sigma$ an integer can be found in Sect. V.2 of [12]. For fractional $\sigma$ a proof along the same lines is possible, using the fact that $H_{\sigma}$ is closed under multiplication (see Theorem 2.1 in [11]).

Returning to the proof of Part (v), we have $F(\varphi) \in H_{\sigma}$, so $\left(\mu^{2}-\Delta\right) \varphi=f$ $-F(\varphi) \in H_{\min (\sigma, s)}$, so $\varphi \in H_{2+\min (\sigma, s)}$.

\section{Proof of Theorem 2 Part (vi)}

If $s \in[-1,1]$ the result is proved in Part (iv). If $s \geqq 1$ then by Part (iv) $\varphi \in \mathscr{I}_{k, 3}$ and $3>d / 2$. The result follows from a finite number of applications of the following bootstrap lemma :

Lemma 5. If $f \in \mathscr{P}_{k, s}$ and $\varphi \in \mathscr{S}_{k, \sigma}$ with $\sigma>d / 2$ then $\varphi \in \mathscr{Y}_{k, 2+\min (s, \sigma)}$.

Proof. Since $\varphi \in \mathscr{Y}_{k, \sigma}$ and $\sigma>d / 2$ it follows from Part (ii) of Lemma 2 that $F(\varphi) \in \mathscr{S}_{k, \sigma}$. Thus $\left(\mu^{2}-\Delta\right) \varphi=f-F(\varphi) \in \mathscr{S}_{k, \min (s, \sigma)}$. Part (i) of the lemma just cited implies that $\varphi \in \mathscr{S}_{k, 2+\min (s, \sigma)}$.

\section{Contraction Estimates}

In this section we suppose that $F \in C(\mathbb{R}), F(0)=0$, and $F$ is nondecreasing, so that $[F(s)-F(t)](s-t) \geqq 0 \forall s, t \in \mathbb{R}$. We begin the proof of (i) in Theorem 3 .

Suppose $\varphi_{1}$ and $\varphi_{2}$ satisfy the field equations with sources $f_{1}$ and $f_{2}$ in $H_{-1}$. Then taking $\varphi_{1}-\varphi_{2}$ as the test function in the field equations for $\varphi_{j}$ yields

$$
\begin{aligned}
& \int \mu^{2}\left(\varphi_{1}-\varphi_{2}\right) \varphi_{j}+\nabla\left(\varphi_{1}-\varphi_{2}\right) \cdot \nabla \varphi_{j} d x \\
& \quad=\left\langle\varphi_{1}-\varphi_{2}, f_{j}-F\left(\varphi_{j}\right)\right\rangle, \quad j=1,2 .
\end{aligned}
$$

Subtracting the $j=2$ equation from the $j=1$ equation yields

$$
\begin{aligned}
& \int \mu^{2}\left(\varphi_{1}-\varphi_{2}\right)^{2}+\left|\prod\left(\varphi_{1}-\varphi_{2}\right)\right|^{2} d x \\
& \quad=\left\langle\varphi_{1}-\varphi_{2}, f_{1}-f_{2}\right\rangle-\left\langle\varphi_{1}-\varphi_{2}, F\left(\varphi_{1}\right)-F\left(\varphi_{2}\right)\right\rangle .
\end{aligned}
$$


The first term on the right is estimated as follows:

$$
\begin{aligned}
& \left|\left\langle\varphi_{1}-\varphi_{2}, f_{1}-f_{2}\right\rangle\right| \leqq\left\|\varphi_{1}-\varphi_{2}\right\|_{H_{1}}\left\|f_{1}-f_{2}\right\|_{H_{-1}} \\
& \left.\quad \leqq\left(1+1 / \mu^{2}\right) \quad \text { [left hand side of }(4.1)\right]^{1 / 2} \quad\left\|f_{1}-f_{2}\right\|_{H_{-1}} .
\end{aligned}
$$

The last term in (4.1) is formally $\int\left(\varphi_{1}-\varphi_{2}\right)\left[F\left(\varphi_{1}\right)-F\left(\varphi_{2}\right)\right] d x$, the integrand being nonnegative. One expects this term to be nonnegative; and once that is proved, (4.1) and (4.2) yield the desired estimate.

Choose $\eta \in C_{0}^{\infty}\left(\mathbb{R}^{d}\right)$ with $\eta \geqq 0$ and $\eta(0)=1$. Let $\eta_{m}(x)=\eta\left(m^{-1} x\right)$. Let $g_{m}: \mathbb{R} \rightarrow \mathbb{R}$ be defined by

$$
g_{m}(s)=\left\{\begin{array}{lll}
s & \text { if } & |g(s)| \leqq m, \\
m s /|s| & \text { if } & |s| \geqq m
\end{array}\right.
$$

Then as $m \rightarrow \infty, \eta_{m}(x) g_{m}\left(\varphi_{j}\right) \rightarrow \varphi_{j}$ in $H_{1}$, so as $m \rightarrow \infty$,

$$
\left\langle\eta_{m}\left[g_{m}\left(\varphi_{1}\right)-g_{m}\left(\varphi_{2}\right)\right], F\left(\varphi_{1}\right)-F\left(\varphi_{2}\right)\right\rangle \rightarrow\left\langle\varphi_{1}-\varphi_{2}, F\left(\varphi_{1}\right)-F\left(\varphi_{2}\right)\right\rangle,
$$

since $F\left(\varphi_{j}\right) \in H_{-1}$. On the other hand, $\eta_{m} g_{m}\left(\varphi_{j}\right) \in L_{\text {compact }}^{\infty}$, and $F\left(\varphi_{i}\right) \in L_{\mathrm{loc}}^{1}$, so

$$
\begin{aligned}
& \left\langle\eta_{m}\left[g_{m}\left(\varphi_{1}\right)-g_{m}\left(\varphi_{2}\right)\right], F\left(\varphi_{1}\right)-F\left(\varphi_{2}\right)\right\rangle \\
& \quad=\int \eta_{m}\left[g_{m}\left(\varphi_{1}\right)-g_{m}\left(\varphi_{2}\right)\right]\left[F\left(\varphi_{1}\right)-F\left(\varphi_{2}\right)\right] d x,
\end{aligned}
$$

and the integrand is nonnegative. It follows that the left hand side is nonnegative, so passing to the limit $m \rightarrow \infty$ the desired inequality $\left\langle\varphi_{1}-\varphi_{2}, F\left(\varphi_{1}\right)-F\left(\varphi_{2}\right)\right\rangle \geqq 0$ follows.

We next prove assertion (ii) of Theorem 3. Formally, the proof is quite simple. This ideas needed to make the argument rigorous are similar to those just presented and those in the $L^{p}$ regularity theorem for $p<\infty$. We present only the formal argument.

Suppose $1<p<\infty$. Subtract the field equation for $\varphi_{2}$ from the equation for $\varphi_{1}$, multiply by $\left|\varphi_{2}-\varphi_{1}\right|^{p-2}\left(\varphi_{2}-\varphi_{1}\right)$, and integrate over $\mathbb{R}^{d}$ to obtain

$$
\begin{aligned}
& \int \mu^{2}\left|\varphi_{2}-\varphi_{1}\right|^{p}+(p-1)\left|\varphi_{2}-\varphi_{1}\right|^{p-2}\left|\nabla\left(\varphi_{2}-\varphi_{1}\right)\right|^{2} \\
& \quad+\left|\varphi_{2}-\varphi_{1}\right|^{p-2}\left(\varphi_{2}-\varphi_{1}\right)\left[F\left(\varphi_{2}\right)-F\left(\varphi_{1}\right)\right] d x \\
& =\int\left|\varphi_{2}-\varphi_{1}\right|^{p-2}\left(\varphi_{2}-\varphi_{1}\right)\left(f_{2}-f_{1}\right) d x .
\end{aligned}
$$

The left hand side is greater than or equal to $\mu^{2}\left\|\varphi_{2}-\varphi_{1}\right\|_{L^{p}}^{p}$, and Hölder's inequality shows that the right hand side is less than or equal to $\left(\left\|\varphi_{2}-\varphi_{1}\right\|_{L^{p}}\right)^{p / q}\left\|f_{2}-f_{1}\right\|_{L^{p}}$. Dividing by $\left(\left\|\varphi_{2}-\varphi_{1}\right\|_{L^{p}}\right)^{p / q}$ completes the derivation.

\section{$V$. Dependence on $\lambda$}

Fix the integer $s$ with $s+2>d / 2$, and suppose that $F \in C^{\infty}(\mathbb{R})$ is nondecreasing and $F(0)=0$. To study the dependence of $\varphi$ on $\lambda$ and $f$, define

$$
G:[0, \infty) \times H_{s+2}\left(\mathbb{R}^{d}\right) \times H_{s}\left(\mathbb{R}^{d}\right) \rightarrow H_{s}\left(\mathbb{R}^{d}\right)
$$

by

$$
G(\lambda, \varphi, f)=\mu^{2} \varphi-\Delta \varphi+\lambda F(\varphi)-f .
$$


A function $\varphi \in H_{s+2}$ (note that $s+2>d / 2$, so $\varphi$ is a continuous function vanishing at infinity) satisfies the field Eq. (1.4) if and only if $G(\lambda, \varphi, f)=0$. The differentiability and analytic dependence results will be proved by applying the implicit function theorem, as in 10.2.1 and 10.2.3 of Dieudonné [13], to this equation. A critical ingredient is Schauder's lemma (see Sect. III.5), which implies that $G$ is an infinitely differentiable map of $[0, \infty) \times H_{s+2} \times H_{s}$ to $H_{s}$, and that the partial derivative of $G$ with respect to $\varphi$ is the linear map

$$
H_{s+2}\left(\mathbb{R}^{d}\right) \ni g \mapsto\left[\mu^{2}-\Delta+\lambda F^{\prime}(\varphi)\right] g \in H_{s}\left(\mathbb{R}^{d}\right) .
$$

By Schauder's lemma again, the function $\lambda F^{\prime}(\varphi) \equiv m(x)$ is in $H_{s+2}$. If $\lambda \geqq 0$ then $m \geqq 0$ since $F^{\prime} \geqq 0$. To apply the implicit function theorem we show that the linear map (5.1) is an isomorphism. That is the content of the next assertion, with $\sigma=s+2$.

Lemma 6. If $m \in H_{\sigma}\left(\mathbb{R}^{d}\right), \sigma>d / 2$, and $m \geqq 0$, then the map $T: g \rightarrow\left(\mu^{2}-\Delta+m\right) g$ is a continuous linear bijection of $H_{\sigma} \rightarrow H_{\sigma-2}$.

Proof. Since $m \in H_{\sigma}$ with $\sigma>d / 2$, Schauder's lemma implies that $g \mapsto m g$ is continuous from $H_{\sigma}$ to itself, and therefore that $T$ is continuous from $H_{\sigma}$ to $H_{\sigma-2}$. Since $m$ is a bounded continuous function, the map $g \mapsto m g$ is also continuous from $H_{0}=L^{2}$ to itself; so by interpolation, it is continuous from $H_{\sigma^{\prime}} \rightarrow H_{\sigma^{\prime}}$ for all $0 \leqq \sigma^{\prime} \leqq \sigma$.

Since $m \geqq 0$,

$$
(T \varphi, \varphi)_{0} \geqq \int \mu^{2} \varphi^{2}+|\nabla \varphi|^{2} d x
$$

for all $\varphi \in H_{1}$, and standard elliptic theory implies that $T$ is an isomorphism of $H_{1} \mapsto H_{-1}$. To complete the proof of the lemma, it suffices to show that if $k \in H_{\sigma-2}$ and $\varphi \in H_{1}$ satisfies $T \varphi=k$ then $\varphi \in H_{\sigma}$; for then $T$ is a $1-1$ continuous linear map of $H_{\sigma} \rightarrow H_{\sigma-2}$ and the open mapping theorem implies the continuity of $T^{-1}$.

Suppose $k \in H_{\sigma-2}, \varphi \in H_{1}$, and $T \varphi=k$. Then a finite number of applications of the following bootstrap lemma leads to the conclusion $\varphi \in H_{\sigma}$.

Lemma 7. If $k \in H_{\tau}\left(\mathbb{R}^{d}\right), \varphi \in H_{\lambda}\left(\mathbb{R}^{d}\right), \sigma \geqq \lambda \geqq 1$, and $T \varphi=k$, then $\varphi \in H_{2+\min (\tau, \lambda)}\left(\mathbb{R}^{d}\right)$.

Proof of Lemma 7. $\left(\mu^{2}-\Delta\right) \varphi=k-m \varphi$ and $m \varphi \in H_{\lambda}$ since multiplication by $m$ maps $H_{\sigma^{\prime}}$ to itself for all $\sigma^{\prime} \in[0, \sigma]$. Thus $k-m \varphi \in H_{\min (\tau, \lambda)}$ so $\varphi \in H_{2+\min (\tau, \lambda)}$.

Given $\left(\lambda_{0}, \varphi_{0}, f_{0}\right) \in[0, \infty) \times H_{s+2} \times H_{s}$ with $G\left(\lambda_{0}, \varphi_{0}, f_{0}\right)=0$, the implicit function theorem implies that for $(\lambda, f)$ close to $\left(\lambda_{0}, f_{0}\right)$ there is a unique $\varphi(\lambda, f)$ close to $\varphi_{0}$ with $G(\lambda, \varphi, f)=0$; and $\varphi$ is a $C^{\infty}$ function from $[0, \infty) \times H_{s}$ to $H_{s+2}$. This completes the proof of Part (i).

We next prove the analyticity result that is Part (ii) of Theorem 4. Fix $s$ as above and $\lambda_{0} \in[0, \infty), \varphi_{0}, f_{0}$ real solutions of $G\left(\lambda_{0}, \varphi_{0}, f_{0}\right)=0$. Let $\mathscr{H}_{s}$ be the space of complex valued functions on $\mathbb{R}^{d}$ whose real and imaginary parts lie in $H_{s}\left(\mathbb{R}^{d}\right)$. Since $F$ is defined on a neighborhood in $\mathbb{C}$ of the real axis, the function $\Gamma$ defined by

$$
\Gamma(\lambda, \varphi)=G\left(\lambda, \varphi, f_{0}\right)
$$


is a $C^{\infty}$ function on a neighborhood $\mathscr{U}$ of $\lambda_{0} \times \varphi_{0}$ in $\mathbb{C} \times \mathscr{H}_{s+2}$ with values in $\mathscr{H}_{s}$. The partial derivative of $\Gamma$ with respect to $\varphi$ is the linear map

$$
\mathscr{H}_{s+2} \ni g \vdash\left[\mu^{2}-\Delta+\lambda_{0} F^{\prime}(\varphi)\right] g \in \mathscr{H}_{s},
$$

and exactly as before this is an isomorphism of $\mathscr{H}_{s+2}$ to $\mathscr{H}_{s^{*}}$. The implicit function theorem implies that there is a neighborhood $\mathscr{F}$ of $\lambda_{0} \in \mathbb{C}$ and a unique $\varphi: \mathscr{H} \rightarrow \mathscr{H}_{s}$ such that $\Gamma[\lambda, \varphi(\lambda)]=0$ and $\varphi: \mathscr{F} \rightarrow \mathscr{H}_{s}$ is infinitely differentiable, the derivative being complex linear. By the characterization of analytic functions as functions differentiable over $\mathbb{C}$ as in 9.10 .1 of [13] it follows that $\varphi$ is analytic on $\mathscr{V}$ with values in $\mathscr{H}_{s}$, so the restriction of $\varphi$ to $\mathscr{F} \cap[0, \infty)$ is real analytic. Since the restriction of $\varphi$ to $[0, \infty)$ yields the real solution of the field equation (uniqueness!) and $\lambda_{0} \in \mathscr{V} \cap[0, \infty)$, this completes the proof of Part (ii).

Finally, we discuss the dependence on $\lambda$ and $f$ when $f$ and $\varphi$ lie in the weighted spaces $\mathscr{S}_{k, s}$ with $s+2>d / 2$. The proof of both the differentiable dependence on $\lambda, f$ and analytic dependence on $\lambda$ are exactly as above once one shows that the map $T$ of Lemma 6 is an isomorphism of $\mathscr{S}_{k, s+2} \rightarrow \mathscr{S}_{k, s^{*}}$

The proof is like the proof of Lemma 6 with a few modifications. First one must show that multiplication by $m$ maps $\mathscr{S}_{k, \sigma} \rightarrow \mathscr{S}_{k, \sigma}$ continuously if $0 \leqq \sigma \leqq s+2$. For $\sigma=0$ this is easy since $m$ is bounded, and for $\sigma=s+2$ it follows from Part (iii) of Lemma 3 (which serves as the natural replacement for Schauder's lemma). It remains to show that $\mathscr{S}_{k, \sigma}$ is an interpolation space between $\mathscr{S}_{k, 0}$ and $\mathscr{S}_{k, s+2}$. To see this, norm $\mathscr{T}_{k, 0}$ by

$$
\|\varphi\|_{\mathscr{S}_{k, 0}}^{2}=\sum_{|\alpha| \leqq k}\left\|x^{\alpha} \varphi\right\|_{L^{2}}^{2}
$$

Then Part (i) of Lemma 3 implies that $\mathscr{F}_{k, \sigma}$ is normed by

$$
\|\varphi\|_{\mathscr{I}_{k, \sigma}}=\left\|\left(\mu^{2}-4\right)^{\sigma / 2} \varphi\right\|_{\mathscr{S}_{k, 0}}^{2}+\|\varphi\|_{\mathscr{S}_{k, 0}}^{2}
$$

(the last term is to make $A$, below, simple). With these choices of norms each $\mathscr{T}_{k, \sigma}$ is a separable Hilbert space, and $\mathscr{S}_{k, \sigma}$ is a dense subset of $\mathscr{S}_{k, 0}$ with continuous inclusion. Consider the intermediate spaces between $\mathscr{F}_{k, 0}$ and $\mathscr{F}_{k, s+2}$ in the framework of Sect. 2.1 of Lions and Magenes [14]. With $X=\mathscr{P}_{k, s+2}, Y=\mathscr{T}_{k, 0}$, and the norms described above, their operator $\Lambda$ is exactly $\left(\mu^{2}-\Delta\right)^{(s+2) / 2}$ and $\left[\mathscr{S}_{k, s+2}, \mathscr{S}_{k, 0}\right]_{\theta}=D\left(\Lambda^{\theta}\right)=\mathscr{S}_{k,(s+2) \theta}$ [again, Part (i) of Lemma 3]. Thus $\mathscr{S}_{k, \sigma}$ $=\left[\mathscr{S}_{k, s+2}, \mathscr{S}_{k, 0}\right]_{\sigma /(s+2)}$

Thus $T$ is a $1-1$ continuous map of $\mathscr{P}_{k, s+2} \rightarrow \mathscr{S}_{k, s}$. To show that it is onto we must show that if $\eta \in \mathscr{P}_{k, s}$ and $\varphi \in H_{s+2}$ satisfies $T \varphi=\eta$ then $\varphi \in \mathscr{P}_{k+2, s}$. This regularity result is analogous to Part (vi) of Theorem 2 and has a similar proof. We omit the details.

\section{References}

1. Boulware,D., Brown,L. : Tree graphs and classical fields. Phys. Rev. 172, 1628-1631 (1968)

2. Williams,D.N.: The Euclidean loop expansion for massive $\lambda \Phi_{4}^{4}$ : Through one loop. Commun. math. Phys. 54, 193-218 (1977)

3. Jackiw, R.: Quantum meaning of classical field theory. Rev. Mod. Phys. 49, 681-706 (1977) 
4. Hopf,E.: Über den funktionalen, insbesondere den analytischen Charakter der Lösungen elliptischer Differentialgleichungen zweiter Ordnung. Math. Z. 34, 194-233 (1931)

5. Moser,J.: A new proof of de Giorgi's theorem concerning the regularity problem for elliptic differential equations. Commun. Pure Appl. Math. 13, 457-468 (1960)

6. Brézis, H., Strauss, W. A. : Semi-linear second-order elliptic equations in $L^{1}$. J. Math. Soc. Japan 25, $566-590(1973)$

7. Schwartz,J.T.: Nonlinear functional analysis, New York: Gordon and Breach 1969

8. Strauss, W.: On weak solutions of semilinear hyperbolic equations. An. Acad. Brasil, Ci. 42, 645 $651(1970)$

9. Treves,F.: Basic linear partial differential equations. New York: Academic Press 1975

10. Gilbarg,D., Trudinger,N.S.: Elliptic partial differential equations of second order. Berlin, Heidelberg, New York: Springer 1977

11. Strichartz, R.: Multipliers on fractional Sobolev spaces. J. Math. Mech. 16, 1031-1060 (1967)

12. Mizohata,S.: Lectures on the Cauchy problem. Bombay: Tata Institute Lecture Notes 1965

13. Dieudonné,J. : Foundations of modern analysis. New York: Academic Press 1969

14. Lions, J.L., Magenes, E.; Non homogeneous boundary value problems and applications, Vol. I (translated by P. Kenneth). New York: Springer 1972

Communicated by J. Glimm

Received May 24, 1978 